\title{
Existence of radial solutions for a $p(x)$-Laplacian Dirichlet problem
}

\author{
Maria Alessandra Ragusa ${ }^{1,2^{*}} \mathbb{D}$, Abdolrahman Razani $^{3}$ and Farzaneh Safari ${ }^{3}$
}

\section{"Correspondence:}

mariaalessandra.ragusa@unict.it 'Dipartimento di Matematica e Informatica, Università di Catania, Catania, Italy

${ }^{2}$ RUDN University, 6

Miklukho-Maklay St, 117198

Moscow, Russia

Full list of author information is available at the end of the article

\begin{abstract}
In this paper, using variational methods, we prove the existence of at least one positive radial solution for the generalized $p(x)$-Laplacian problem

$$
-\Delta_{p(x)} u+R(x) u^{p(x)-2} u=a(x)|u|^{q(x)-2} u-b(x)|u|^{r(x)-2} u
$$

with Dirichlet boundary condition in the unit ball in $\mathbb{R}^{N}$ (for $N \geq 3$ ), where $a, b, R$ are radial functions.

MSC: Primary 35J15; secondary 58E30

Keywords: Radial solution; $p(x)$-Laplacian; Dirichlet boundary condition; Variational principle
\end{abstract}

\section{Introduction}

The study of differential equations and variational problems with nonstandard $p(x)$ growth conditions (or nonstandard $(p, q)$-growth conditions) is an attractive topic and has been the object of considerable attention in recent years (see [1]). The reasons for such an interest are as follows: 1) Physically, it relies on the fact that they model phenomena arising from various fields such as the motion of electrorheological fluids, which are characterized by their ability to drastically change their mechanical properties under the influence of an exterior electromagnetic field, the thermo-convective flows of non-Newtonian fluids, and the image processing; 2) Mathematically, it relies on the fact that the standard mathematical techniques are not adequate to study these problems and they need new techniques. This may be the central development of mathematical ideas in active areas of pure mathematics, which have had a decisive interaction with PDEs (such as [2-14]).

The aim of this paper is to prove the existence of at least one positive radial solution belonging to the space $W_{0}^{1, p(\cdot)}(B) \cap L_{a}^{q(\cdot)}(B) \cap L_{b}^{r(\cdot)}(B)$ for the problem

$$
\begin{cases}-\Delta_{p(x)} u+R(x) u^{p(x)-2} u=a(x)|u|^{q(x)-2} u-b(x)|u|^{r(x)-2} u, & x \in B, \\ u>0, & x \in B, \\ u=0, & x \in \partial B,\end{cases}
$$

(c) The Author(s) 2021. This article is licensed under a Creative Commons Attribution 4.0 International License, which permits use, sharing, adaptation, distribution and reproduction in any medium or format, as long as you give appropriate credit to the original author(s) and the source, provide a link to the Creative Commons licence, and indicate if changes were made. The images or other third party material in this article are included in the article's Creative Commons licence, unless indicated otherwise in a credit line to the material. If material is not included in the article's Creative Commons licence and your intended use is not permitted by statutory regulation or exceeds the permitted use, you will need to obtain permission directly from the copyright holder. To view a copy of this licence, visit http://creativecommons.org/licenses/by/4.0/. 
where $\Delta_{p(x)}:=\operatorname{div}\left(|\nabla|^{p(x)-2} \nabla\right), B$ is the unit ball centered at the origin in $\mathbb{R}^{N}, N \geq 3, p, q, r \in$ $C_{+}(B), R$ is a positive radial function (introduced in Theorem 1.1), and finally

$$
a(x)=\theta(|x|) \quad \text { and } \quad b(x)=\xi(|x|),
$$

where $\theta, \xi \in L^{\infty}(0,1)$ are such that $\theta$ is a positive nonconstant radially nondecreasing function, and $\xi$ is a nonnegative radially nonincreasing function. The statement of the main result of this paper is as follows.

Theorem 1.1 Let $B$ be the unit ball centered at the origin in $\mathbb{R}^{N}, N \geq 3$, and let $p, q, r \in$ $C_{+}(B)$ be such that $p^{+}, r^{+}<q^{-}, r(x)<q(x)$ a.e. in $B$, and $p(x)<q(x)<p^{*}(x)$ a.e. in $B$, where $p^{*}(x)$ is the Sobolev conjugate of $p(x)$. Assume that $a, b \in L^{\infty}(B)$ are nonconstant radial functions as in (2). The $p(x)$-Laplacian Dirichlet problem (1) admits at least one radial increasing solution in $V=W_{0}^{1, p(\cdot)}(B) \cap L_{a}^{q(\cdot)}(B) \cap L_{b}^{r \cdot(\cdot)}(B)$ if one of the following conditions is true for a radial function $R: B \rightarrow[0,+\infty)$ :

(i) $R(x)=\alpha$, where $\alpha \geq 0$ is a real constant.

(ii) $R \in L^{\infty}(B)$ and $\mu>0$, where $\mu=\inf _{u \in W_{0}^{1, p(\cdot)}(B) \backslash\{0\}} \frac{\int_{B}|\nabla u|^{p(x)} d \xi}{\int_{B}|u|^{p(x)} d x}$.

Before verifying our approach, we prepare some preliminaries. From now on we assume that $B$ is the unit ball centered at the origin in $\mathbb{R}^{N}, N \geq 3$, and we set

$$
p^{-}=\inf _{x \in B} p(x) \quad \text { and } \quad p^{+}=\sup _{x \in B} p(x)
$$

where $p \in C_{+}(\bar{B})=\left\{g \in C(\bar{B}): g^{-}>1\right\}$. The generalized Lebesgue space $L^{p(\cdot)}(B)$ is the collection of all measurable functions $u$ on $B$ such that $\int_{B}|u(x)|^{p(x)} d x<+\infty$ with the norm

$$
|u|_{p(\cdot)}=\inf \left\{\lambda>0: \int_{B}\left|\frac{u(x)}{\lambda}\right|^{p(x)} d x \leq 1\right\}
$$

For any $u \in L^{p(\cdot)}(B)$ and $v \in L^{p^{\prime} \cdot(\cdot)}(B)$, where $L^{p^{\prime}(\cdot)}(B)$ is the conjugate space of $L^{p(\cdot)}(B)$, we have the Hölder-type inequality

$$
\left|\int_{B} u v d x\right| \leq\left(\frac{1}{p^{-}}+\frac{1}{p^{\prime-}}\right)|u|_{p(\cdot)}|v|_{p^{\prime}(\cdot)}
$$

The following proposition is well known in Lebesgue spaces with variational exponent (e.g., see [15, Proposition 2.7]).

Proposition 1.2 For any $u \in L^{p(x)}(B)$, we have

$$
|u|_{p(x)}^{p^{-}} \leq \int_{B}|u(x)|^{p(x)} d x \leq|u|_{p(x)}^{p^{+}}
$$

if $|u|_{p(x)} \geq 1$ and

$$
|u|_{p(x)}^{p^{+}} \leq \int_{B}|u(x)|^{p(x)} d x \leq|u|_{p(x)}^{p^{-}}
$$

if $|u|_{p(x)}<1$. 
Normally, the Sobolev space associated with $L^{p(\cdot)}(B)$ is defined as follows:

$$
W^{1, p(\cdot)}(B)=\left\{u: B \rightarrow \mathbb{R}: u,|\nabla u| \in L^{p(\cdot)}(B)\right\}
$$

endowed with the norm

$$
\|u\|_{p(\cdot)}=|\nabla u|_{p(\cdot)}+|u|_{p(\cdot)},
$$

where $\nabla u=\left(\frac{\partial u}{\partial x_{1}}(x), \ldots, \frac{\partial u}{\partial x_{N}}(x)\right)$ is the gradient of $u$ at $x=\left(x_{1}, \ldots, x_{n}\right)$ and, as usual, $|\nabla u|=\left(\sum_{i=1}^{N}\left|\frac{\partial u}{\partial x_{i}}\right|^{2}\right)^{\frac{1}{2}}$. We set $W_{0}^{1, p(\cdot)}(B)=\overline{\left(C_{0}^{\infty}(B),\|\cdot\|_{p(\cdot)}\right)}$. We know that the spaces $L^{p(\cdot)}(B), W^{1, p(\cdot)}(B)$ and $W_{0}^{1, p(\cdot)}(B)$ are reflexive, uniformly convex, and separable Banach spaces.

The following theorem is [16, Theorem 2.8].

Theorem 1.3 Let $\Omega$ be a bounded smooth set in $\mathbb{R}^{N}$, and let $p, q \in C_{+}(\bar{\Omega})$. Then

$$
L^{q(\cdot)}(\Omega) \hookrightarrow L^{p(\cdot)}(\Omega)
$$

if and only if $p(x) \leq q(x)$ for a.e. $x \in \Omega$.

As a consequence of Theorem 1.3, we have

$$
W^{1, q(\cdot)}(\Omega) \hookrightarrow W^{1, p(\cdot)}(\Omega)
$$

if $p(x) \leq q(x)$ for a.e. $x \in \Omega$.

The following proposition is proved in [17] (also, see [18, Theorem 8.2.4]).

Proposition 1.4 Let $\Omega$ be a bounded smooth set in $\mathbb{R}^{N}$, and let $p, q \in C_{+}(\bar{\Omega})$. Then

(i) If $q(x)<p^{*}(x)$ for any $x \in \bar{\Omega}$, then the embedding $W^{1, p(x)}(\Omega) \hookrightarrow L^{q(x)}(\Omega)$ is compact and continuous, where

$$
p^{*}(x)= \begin{cases}\frac{N p(x)}{N-p(x)}, & p(x)<N, \\ +\infty, & p(x) \geq N .\end{cases}
$$

(ii) There is a constant $C>0$ such that

$$
|u|_{p(x)} \leq C|\nabla u|_{p(x)} \quad \text { for all } u \in W_{0}^{1, p(x)}(\Omega)
$$

Remark 1.5 By part (ii) of Proposition 1.4 we can see that $\|u\|_{*}=|\nabla u|_{p(x)}$ and $\|u\|_{p(x)}$ are equivalent norms on $W_{0}^{1, p(x)}(B)$.

Lemma 1.6 Let $R: B \rightarrow[0,+\infty)$ satisfy one of the conditions of Theorem 1.1, and define

$$
\varrho(u)=\int_{B}|\nabla u|^{p(x)}+R(x)|u|^{p(x)} d x .
$$


Then there exists a constant $M>0$ such that

$$
\|u\|_{*}^{\check{p}} \leq \varrho(u) \leq M\|u\|_{*}^{\hat{p}}
$$

for all $u \in W_{0}^{1, p(\cdot)}(B)$, where

$$
\check{p}=\left\{\begin{array}{ll}
p^{+}, & \|u\|_{*}<1, \\
p^{-}, & \|u\|_{*} \geq 1,
\end{array} \quad \hat{p}= \begin{cases}p^{-}, & \|u\|_{*}<1, \\
p^{+}, & \|u\|_{*} \geq 1 .\end{cases}\right.
$$

Proof First, suppose that $\|u\|_{*}=|\nabla u|_{p(\cdot)} \geq 1,|u|_{p(\cdot)} \geq 1$. By Proposition 1.2 we have

$$
\|u\|_{*}^{\check{p}}=|\nabla u|_{p(\cdot)}^{p^{-}} \leq \varrho(u) \leq|\nabla u|_{p(\cdot)}^{p^{+}}+C^{\prime}|u|_{p(\cdot)}^{p^{+}}
$$

So, according to the Poincaré inequality (Proposition 1.4, part (ii)),

$$
\|u\|_{*}^{p^{-}} \leq \varrho(u) \leq\left(1+C^{\prime \prime}\right)\|u\|_{*}^{p^{+}}
$$

Now suppose that $\|u\|_{*}=|\nabla u|_{p(\cdot)} \geq 1,|u|_{p(\cdot)}<1$. Then we have

$$
\|u\|_{*}^{\check{p}}=|\nabla u|_{p(\cdot)}^{p^{-}} \leq \varrho(u) \leq|\nabla u|_{p(\cdot)}^{p^{+}}+C^{\prime}|u|_{p(\cdot)}^{p^{-}}
$$

Since $|u|_{p(\cdot)}^{p^{-}} \leq C_{1}\|u\|_{*}^{p^{-}} \leq C_{1}\|u\|_{*}^{p^{+}}$, this implies that

$$
\|u\|_{*}^{p^{-}} \leq \varrho(u) \leq\left(1+C^{\prime} C_{1}\right)\|u\|_{*}^{p^{+}} .
$$

Now assume that $\|u\|_{*}=|\nabla u|_{p(\cdot)}<1,|u|_{p(\cdot)} \geq 1$. Then by Proposition 1.2

$$
\|u\|_{*}^{\hat{p}}=|\nabla u|_{p(\cdot)}^{p^{+}} \leq \varrho(u) \leq|\nabla u|_{p(\cdot)}^{p^{-}}+C^{\prime}|u|_{p(\cdot)}^{p^{+}}
$$

By the Poincaré inequality we have

$$
\|u\|_{*}^{p^{+}} \leq \varrho(u) \leq\left(1+C C^{\prime}\right)\|u\|_{*}^{p^{+}} \leq\left(1+C C^{\prime}\right)\|u\|_{*}^{p^{-}} .
$$

Finally, assume that $\|u\|_{*}=|\nabla u|_{p(\cdot)}<1,|u|_{p(\cdot)}<1$. Then

$$
\|u\|_{*}^{\hat{p}}=|\nabla u|_{p(\cdot)}^{p^{+}} \leq \varrho(u) \leq|\nabla u|_{p(\cdot)}^{p^{-}}+C^{\prime}|u|_{p(\cdot)}^{p^{-}} .
$$

As before, $|u|_{p(\cdot)}^{p^{-}} \leq C\|u\|_{*}^{p^{-}}$, and thus

$$
\|u\|_{*}^{p^{+}} \leq \varrho(u) \leq\left(1+C C^{\prime}\right)\|u\|_{*}^{p^{-}} .
$$

By inequalities (7)-(10) and the definitions of $\breve{p}$ and $\hat{p}$ in (6) we get inequality (5).

Now we recall some notations and results to be used further. For radial functions $a, b \in$ $L^{\infty}(B)$ given by (2), we consider the spaces

$$
L_{a}^{q(\cdot)}(B)=\left\{u: B \rightarrow \mathbb{R}: \int_{B} a(x)|u|^{q(x)} d x<\infty\right\}
$$


with the norms $|u|_{a, q(\cdot)}=\inf \left\{\lambda>0: \int_{B} a(x)\left|\frac{u(x)}{\lambda}\right|^{q(x)} d x \leq 1\right\}$. Similarly,

$$
L_{b}^{r(\cdot)}(B)=\left\{u: B \rightarrow \mathbb{R}: \int_{B} b(x)|u|^{r(x)} d x<\infty\right\}
$$

with the norms $|u|_{b, r(\cdot)}=\inf \left\{\lambda>0: \int_{B} b(x)\left|\frac{u(x)}{\lambda}\right|^{r(x)} d x \leq 1\right\}$.

Definition 1.7 (Subdifferential) Let $V$ be a real Banach space, and let $V^{*}$ be its topological dual with pairing between $V$ and $V^{*}$ denoted by $\langle\cdot, \cdot\rangle$. Let $\Psi: V \rightarrow(-\infty,+\infty]$ be a proper convex function, and let $2^{V^{*}}$ be the set of all subsets of $V^{*}$. The subdifferential $\partial \Psi: V \rightarrow$ $2^{V^{*}}$ of $\Psi$ is defined as the following set-valued operator:

$$
\partial \Psi(u)=\left\{u^{*} \in V^{*}: \Psi(v) \geq \Psi(u)+\left\langle u^{*}, v-u\right\rangle \text { for all } v \in V\right\}
$$

for $u \in \operatorname{Dom}(\Psi)=\{v \in V ; \Psi(v)<\infty\}$, and $\partial \Psi(u)=\emptyset$ if $u \notin \operatorname{Dom}(\Psi)$.

Note that if $\Psi$ is Gâteaux differentiable at $u$ with its derivative denoted by $D \Psi(u)$, then $\partial \Psi(u)$ is a singleton. In this case, $\partial \Psi(u)=\{D \Psi(u)\}$.

Lemma 1.8 ([19, Theorem 1.5.3]) Let $V$ be a reflexive Banach space, and let $I: V \rightarrow \mathbb{R}$ be a continuous convex functional. Then I is weakly lower semicontinuous.

The following is the Weierstrass theorem (see [19, Theorem 1.5.6.]).

Theorem 1.9 Let $V$ be a reflexive Banach space, and let $I: V \rightarrow \mathbb{R}$ be a weakly lower semicontinuous coercive functional. Then I has a global minimum point.

Now we state the definition of a critical point of the functional.

Definition 1.10 (Critical point) Let $V$ be a real Banach space, let $\Phi \in C^{1}(V, \mathbb{R})$, and let $\Psi: V \rightarrow(-\infty,+\infty$ ] be a proper (i.e., Dom $\Psi \neq \emptyset)$, convex, and lower semicontinuous function. Let $K \subset V$ be a weakly convex closed set. Define the function $\Psi_{K}: V \rightarrow(-\infty,+\infty]$ by

$$
\Psi_{K}(u):= \begin{cases}\Psi(u), & u \in K, \\ +\infty, & u \notin K .\end{cases}
$$

Consider the functional

$$
I_{K}:=\Psi_{K}-\Phi
$$

We say that $u \in V$ is a critical point of $I_{K}$ if $D \Phi(u) \in \partial \Psi_{K}(u)$ or, equivalently, it satisfies in the inequality

$$
\langle D \Phi(u), u-v\rangle+\Psi_{K}(v)-\Psi_{K}(u) \geq 0 \quad \text { for all } v \in V .
$$

Note that a global minimum point is a critical point. 
Definition 1.11 (PS compactness condition) We say that $I_{K}$ (12) satisfies the Palais-Smale (PS) compactness condition if for any sequence $\left\{u_{n}\right\}$ such that

- $I_{K}\left(u_{n}\right) \rightarrow c \in \mathbb{R}$ and

- $\left\langle D \Phi\left(u_{n}\right), u_{n}-v\right\rangle+\Psi_{K}(v)-\Psi_{K}\left(u_{n}\right) \geq-\epsilon_{n}\left\|v-u_{n}\right\|$

for all $v \in V$ as $\epsilon_{n} \rightarrow 0$, then $\left\{u_{n}\right\}$ possesses a convergent subsequence.

The following mountain pass geometry (MPG) theorem was proved in [20].

Theorem 1.12 Suppose that $I_{K}: V \rightarrow(-\infty,+\infty]$ is of the form (12) and satisfies the PS compactness condition and the following conditions are satisfied:

(i) $I_{K}(0)=0$,

(ii) there exists $e \in V$ such that $I_{K}(e) \leq 0$,

(iii) there exists a positive constant $\rho$ such that $I_{K}(u)>0$ if $\|u\|=\rho$.

Then $I_{K}$ has a critical value $c \leq \rho$ characterized by

$$
c=\inf _{g \in \Gamma \in[0,1]} I_{K}(g(t))
$$

where $\Gamma=\{g \in C([0,1], V): g(0)=0, g(1)=e\}$.

The next theorem is a fact in [21, Problem 127, p. 81].

Theorem 1.13 Let $\left\{u_{n}\right\}$ be a sequence of nondecreasing (continuous or discontinuous) real functions on $[c, d]$ that converges pointwise to a continuous function $u:[c, d] \rightarrow \mathbb{R}$. Then the convergence is uniform.

Proof Let $u:=\lim _{n \rightarrow \infty} u_{n}$, and let $\varepsilon>0$. We have to show that for $n$ large enough,

$$
\left|u_{n}(x)-u(x)\right| \leq \varepsilon \quad \text { for all } x \in[c, d] .
$$

Since $u$ is assumed to be continuous, it is uniformly continuous on the compact interval $[c, d]$. So there is a subdivision $c=x_{0}<x_{1}<\cdots<x_{k}=d$ of $[c, d]$ such that the oscillation of $u$ on each interval $\left[x_{i}, x_{i+1}\right]$ is less than $\frac{\varepsilon}{2}$. Since $u_{n}\left(x_{i}\right) \rightarrow u\left(x_{i}\right)$ as $n \rightarrow \infty$ for $i=0, \ldots, k$, there is $M$ such that if $n \geq M$, then

$$
\left|u_{n}\left(x_{i}\right)-u\left(x_{i}\right)\right| \leq \frac{\varepsilon}{2} \quad \text { for } i=0, \ldots, k .
$$

Let us check that $\left|u_{n}(x)-u(x)\right| \leq \varepsilon$ for every $n \geq M$ and all $x \in[c, d]$. Fix $n \geq M$ and take any $x \in[c, d]$. We can choose $i$ such that $x \in\left[x_{i}, x_{i+1}\right]$. Since the functions $u_{n}$ are nondecreasing, we have

$$
u_{n}\left(x_{i}\right) \leq u_{n}(x) \leq u_{n}\left(x_{i+1}\right) .
$$

Since $\left|u_{n}\left(x_{i}\right)-u\left(x_{i}\right)\right|$ and $\left|u_{n}\left(x_{i+1}\right)-u\left(x_{i+1}\right)\right|$ are not greater than $\frac{\varepsilon}{2}$, it follows that

$$
u\left(x_{i}\right)-\frac{\varepsilon}{2} \leq u_{n}(x) \leq u\left(x_{i+1}\right)+\frac{\varepsilon}{2} .
$$


Moreover, since the oscillation of $u$ on $\left[x_{i}, x_{i+1}\right]$ is less than $\frac{\varepsilon}{2}$ and since $x \in\left[x_{i}, x_{i+1}\right]$, we also have $u\left(x_{i}\right) \geq u(x)-\frac{\varepsilon}{2}$ and $u\left(x_{i+1}\right) \leq u(x)+\frac{\varepsilon}{2}$. Altogether, this gives

$$
u(x)-\frac{2 \varepsilon}{2} \leq u_{n}(x) \leq u(x)+\frac{2 \varepsilon}{2},
$$

which concludes the proof.

\section{$2 p(x)$-Laplacian equation}

Here we recall the variational principle established in [22].

Definition 2.1 (Pointwise invariance condition) Let $V, \Psi, \Phi, K$ be defined as in Definition 1.10. We say that the triple $(\Psi, \Phi, K)$ satisfies the pointwise invariance condition at a point $u \in V$ if there exist a convex Gâteaux differentiable function $G: V \rightarrow \mathbb{R}$ and a point $v \in K$ such that

$$
D \Psi(v)+D G(v)=D \Phi(u)+D G(u) .
$$

Theorem 2.2 Let $V$ be a reflexive Banach space, and let $K$ be a weakly closed convex subset of $V$. Let $\Psi: V \rightarrow(-\infty,+\infty]$ be a convex lower semicontinuous function that is Gâteaux differentiable on $K$, and let $\Phi \in C^{1}(V, \mathbb{R})$. Assume that the following two conditions hold:

(i) The functional $I_{K}: V \rightarrow(-\infty,+\infty]$ defined by $I_{K}(w)=\Psi_{K}(w)-\Phi(w)$, where $\Psi_{K}$ is defined in (11), has a critical point $u \in V$ as in Definition 1.10,

(ii) the triple $\left(\Psi_{K}, \Phi, K\right)$ satisfies the pointwise invariance condition at the point $u$.

Then $u \in K$ is a solution of the equation

$$
D \Psi(u)=D \Phi(u) .
$$

To apply Theorem 2.2, consider the reflexive Banach space

$$
V=W_{0, r a d}^{1, p(\cdot)}(B) \cap L_{a}^{q(\cdot)}(B) \cap L_{b}^{r(\cdot)}(B)
$$

endowed with the norm

$$
\|u\|_{V}=\|u\|_{*}+|u|_{a, q(\cdot)}+|u|_{b, r(\cdot)},
$$

where

$$
W_{0, r a d}^{1, p(\cdot)}(B)=\left\{u \mid u \in W_{0}^{1, p(\cdot)}(B) \text {, and } u \text { is radial }\right\} .
$$

Definition 2.3 (Weak solution) Let $p, q, r \in C_{+}(B), a, b \in L^{\infty}(B)$, and $R$ be given as in Theorem 1.1, and let $V$ be the space as in (14). We say that $u \in V$ is a (weak) solution of problem (1) if $u$ is increasing and satisfies the Dirichlet boundary conditions and also if the following equality is true for all $w \in V$ :

$$
\begin{aligned}
& \int_{B}|\nabla u|^{p(x)-2} \nabla u \nabla w d x+\int_{B} R(x)|u|^{p(x)-2} u w d x \\
& +\int_{B} b(x)|u|^{r(x)-2} u w d x=\int_{B} a(x)|u|^{q(x)-2} u w d x
\end{aligned}
$$


To prove the claim, we consider the Euler-Lagrange energy functional corresponding to problem (1)

$$
\begin{aligned}
I(u):= & \frac{1}{p(x)}\left(\int_{B}|\nabla u(x)|^{p(x)}+R(x)|u(x)|^{p(x)} d x\right) \\
& +\frac{1}{r(x)} \int_{B} b(x)|u|^{r(x)} d x-\frac{1}{q(x)} \int_{B} a(x)|u|^{q(x)} d x
\end{aligned}
$$

over the convex closed set

$$
K=\{u \in V: u \geq 0, u \text { is an increasing radial function }\} .
$$

Concerning Theorem 2.2, we define $\psi, \varphi: V \rightarrow \mathbb{R}$ by

$$
\psi(u)=\frac{1}{p(x)} \int_{B}|\nabla u|^{p(x)}+R(x)|u|^{p(x)} d x+\frac{1}{r(x)} \int_{B} b(x)|u|^{r(x)} d x
$$

and

$$
\varphi(u)=\frac{1}{q(x)} \int_{B} a(x)|u|^{q(x)} d x .
$$

Notice that $\psi$ is a proper, convex, and lower semicontinuous and $D \varphi(u)=a(x)|u|^{q-2} u$. Therefore $\varphi$ is a $C^{1}$ - function on the space $V$. Let us introduce the functional $I_{K}: V \rightarrow$ $(-\infty,+\infty]$ defined by

$$
I_{K}(u)=\psi_{K}(u)-\varphi(u),
$$

where $\psi_{K}$ is as in (11). Note that $I_{K}=I=\psi-\varphi$ on $K$. We prove Theorem 1.1 in two steps.

Step 1. We show that $I_{K}=\psi_{K}-\varphi$ has a critical point in $K$. For this reason, we use the MPG theorem (Theorem 1.12), in which we will need the following lemma.

Lemma 2.4 There exists $C>0$ such that

$$
\|u\|_{*} \leq\|u\|_{V} \leq C\|u\|_{*} \quad \text { for all } u \in K
$$

Proof Using the Hölder inequality and Remark 1.5, we get

$$
\begin{aligned}
\|u\|_{*} \leq\|u\|_{V} & =\|u\|_{*}+|u|_{a, q(\cdot)}+|u|_{b, r(\cdot)} \\
& \leq\|u\|_{*}+|a|_{\infty}|u|_{q(\cdot)}+|b|_{\infty}|u|_{r(\cdot)} \\
& \leq\|u\|_{*}+c_{1}|a|_{\infty}\|u\|_{q(\cdot)}+c_{2}|b|_{\infty}\|u\|_{r(\cdot)} \\
& \leq\left(1+C_{1}+C_{2}\right)\|u\|_{*} .
\end{aligned}
$$

Lemma 2.5 Let the functional $I_{K}: V \rightarrow \mathbb{R}$ be defined by (19). Then $I_{K}$ satisfies the PS compactness condition in $K$. 
Proof Suppose that $\left\{u_{n}\right\}$ is a sequence in $K$ such that $I_{K}\left(u_{n}\right) \rightarrow c \in \mathbb{R}, \epsilon_{n} \rightarrow 0$, and

$$
\left\langle D \varphi\left(u_{n}\right), u_{n}-v\right\rangle+\psi_{K}(v)-\psi_{K}\left(u_{n}\right) \geq-\epsilon_{n}\left\|v-u_{n}\right\|_{V}
$$

for all $v \in V$. We show that $\left\{u_{n}\right\}$ has a convergent subsequence in $V$. First, notice that $u_{n} \in \operatorname{Dom}(\psi)$. Then

$$
I_{K}\left(u_{n}\right)=\psi_{K}\left(u_{n}\right)-\varphi\left(u_{n}\right) \rightarrow c \quad \text { as } n \rightarrow \infty .
$$

Thus, for large values of $n$, we have

$$
\psi_{K}\left(u_{n}\right)-\varphi\left(u_{n}\right) \leq 1+c .
$$

Furthermore,

$$
\left\langle D \varphi\left(u_{n}\right), u_{n}\right\rangle=\int_{B} a(x) u_{n}(x)^{q(x)} d x \geq q^{-} \varphi\left(u_{n}\right) .
$$

Now consider the function $g(s)=s^{r^{+}}-q^{-}(s-1)-1$ on the interval $(1,+\infty)$. Set $s^{*}=\left(\frac{q^{-}}{r^{+}}\right)^{\frac{1}{r^{+}-1}}$. Obviously, $g(s)<0$ for all $s \in\left(1, s^{*}\right)$. We choose such a number $s$. So we have $s>1$ and $s^{r^{+}}-1<q^{-}(s-1)$. Setting $v=s u_{n}$ in (20), we can see that

$$
(1-s)\left\langle D \varphi\left(u_{n}\right), u_{n}\right\rangle+\left(s^{r^{+}}-1\right) \psi_{K}\left(u_{n}\right) \geq-\epsilon_{n}(s-1)\left\|u_{n}\right\|_{V} .
$$

Therefore

$$
(s-1) q^{-} \varphi\left(u_{n}\right)-\left(s^{r^{+}}-1\right) \psi_{K}\left(u_{n}\right) \leq \epsilon_{n}(s-1)\left\|u_{n}\right\|_{V} \leq C \epsilon_{n}\left\|u_{n}\right\|_{V} .
$$

Take $\alpha>0$ such that

$$
\frac{1}{q^{-}(s-1)}<\alpha<\frac{1}{s^{r^{+}}-1} .
$$

Multiplying (24) by $\alpha$ and summing it up with (21), we get

$$
\begin{aligned}
& {\left[\alpha q^{-}(s-1)-1\right] \frac{1}{q^{+}} \int_{B} a(x)\left|u_{n}\right|^{q(x)} d x+\frac{\left[\alpha\left(1-s^{r^{+}}\right)+1\right]}{p(x)} \varrho\left(u_{n}\right)} \\
& +\frac{1}{r^{+}} \int_{B} b(x)|u|^{r(x)} d x \leq c+1+\alpha C\left\|u_{n}\right\|_{V},
\end{aligned}
$$

where $\varrho$ is defined as in (4). Then

$$
\begin{aligned}
& {\left[\alpha q^{-}(s-1)-1\right] \frac{1}{q^{+}} \int_{B} a(x)\left|u_{n}\right|^{q(x)} d x+\frac{\left[\alpha\left(1-s^{r^{+}}\right)+1\right]}{p^{+}}\left\|u_{n}\right\|_{*}^{\check{p}}} \\
& \quad \leq c+1+\alpha C\left\|u_{n}\right\|_{V} .
\end{aligned}
$$

So by Lemma (2.4) there exists $C^{\prime}>0$ such that

$$
\left\|u_{n}\right\|_{*}^{\check{p}} \leq C^{\prime}\left(1+\left\|u_{n}\right\|_{*}\right)
$$


Therefore $\left\{u_{n}\right\}$ is a bounded sequence in the reflexive space $W_{0, \text { rad }}^{1, p(\cdot)}(B)$. The standard results in Sobolev space imply that there exists $\bar{u} \in W_{0, \text { rad }}^{1, p(\cdot)}(B)$ such that, up to subsequences, for $s \in C_{+}(B)$,

- $u_{n} \rightarrow \bar{u}$ in $W_{0, \text { rad }}^{1, p(\cdot)}(B)$;

- $u_{n} \rightarrow \bar{u}$ in $L^{s(\cdot)}(B), s(x)<p^{*}(x)$ a.e.;

- $u_{n}(x) \rightarrow \bar{u}(x)$ a.e. in $B$

- there exist $w_{s} \in L^{s(\cdot)}(B)$ such that $\left|u_{n}(x)\right| \leq w_{s}(x)$ a.e. in $B$ and for all $n \in \mathbb{N}$ where $s(x)<p^{*}(x)$.

Also, notice that every $u_{n}$ is radial, so $\bar{u}$ is radial, too. Moreover, $\bar{u} \in K$. Therefore $\bar{u} \in$ $\operatorname{Dom}(\psi)$. Now in (20) replace $v$ with $\bar{u}$ :

$$
\begin{gathered}
-\int_{B} a(x)\left|u_{n}\right|^{q(x)-1}\left(\bar{u}-u_{n}\right) d x+\frac{1}{p(x)} \varrho(\bar{u})-\frac{1}{p(x)} \varrho\left(u_{n}\right) \\
+\frac{1}{r(x)} \int_{B} b(x)\left(|\bar{u}|^{r(x)}-\left|u_{n}\right|^{r(x)}\right) d x \geq-\epsilon_{n}\left\|\bar{u}-u_{n}\right\|_{V},
\end{gathered}
$$

where $\varrho$ is defined as in (4). On the one hand, we have

$$
\left.\left|\int_{B} a(x)\right| u_{n}\right|^{q(x)-1}\left(\bar{u}-u_{n}\right) d x\left|\leq \int_{B} a(x) w_{q-1}^{q(x)-1}(x)\right| \bar{u}-u_{n} \mid d x
$$

and $u_{n} \rightarrow \bar{u}$ in $L^{r(x)}(B)$. On the other hand, $\left\{u_{n}\right\} \subset K$, and so $\left\{\left|\bar{u}-u_{n}\right|\right\}$, as $\left\{u_{n}\right\}$ is a sequence consisting of nondecreasing functions that converges to the continuous zero function. Now thanks to Theorem 1.13, the right-hand side of the latter inequality goes to zero. Therefore, passing to limits, we have

$$
\limsup _{n \rightarrow \infty} \varrho\left(u_{n}\right) \leq \varrho(\bar{u}) \leq\|\bar{u}\|_{*}^{\hat{p}} .
$$

Claim: There exists a positive constant $C$ such that $\varrho\left(u_{n}-\bar{u}\right) \leq C \varrho(\bar{u})$, and then $\left\{\varrho\left(u_{n}-\bar{u}\right)\right\}$ is a bounded sequence.

Firstly, assume that $\left|\nabla u_{n}\right| \leq|\nabla \bar{u}|$, so that $\left|\nabla u_{n}-\nabla \bar{u}\right| \leq 2|\nabla \bar{u}|$. Then

$$
\int_{B}\left|\nabla u_{n}-\nabla \bar{u}\right|^{p(x)} d x \leq 2^{p^{+}} \int_{B}|\nabla \bar{u}|^{p(x)} d x \leq c \varrho(\bar{u})
$$

If $\left|\nabla u_{n}\right| \geq|\nabla \bar{u}|$ and thus $\left|\nabla u_{n}-\nabla \bar{u}\right| \leq 2\left|\nabla u_{n}\right|$, then by inequality (25)

$$
\int_{B}\left|\nabla u_{n}-\nabla \bar{u}\right|^{p(x)} d x \leq 2^{p^{+}} \int_{B}\left|\nabla u_{n}\right|^{p(x)} d x \leq 2^{p^{+}} \varrho\left(u_{n}\right) \leq c \varrho(\bar{u}) .
$$

Now assume that $\left|u_{n}\right| \leq|\bar{u}|$. The by the inequality $\left|u_{n}-\bar{u}\right| \leq\left|u_{n}\right|+\bar{u}|\leq 2| \bar{u} \mid$ we have

$$
\int_{B}\left|u_{n}-\bar{u}\right|^{p(x)} d x \leq 2^{p^{+}} \int_{B}|\bar{u}|^{p(x)} d x \leq c^{\prime} \varrho(\bar{u}) .
$$

Ultimately, if $\left|u_{n}\right| \geq|\bar{u}|$ and thus $\left|u_{n}-\bar{u}\right| \leq 2\left|u_{n}\right|$, then from inequality (25) we have

$$
\int_{B}\left|u_{n}-\bar{u}\right|^{p(x)} d x \leq 2^{p^{+}} \int_{B}\left|u_{n}\right|^{p(x)} d x \leq 2^{p^{+}} \varrho\left(u_{n}\right) \leq c^{\prime} \varrho(\bar{u}) .
$$


Therefore $\varrho\left(u_{n}-\bar{u}\right) \leq C \varrho(\bar{u})$, where $C=c+c^{\prime}|R|_{\infty}$, and thus our claim is proved. In fact, we have $u_{n}, \bar{u} \in W_{0, r a d}^{1, p(\cdot)}(B)$ and $u_{n}(x) \rightarrow \bar{u}(x)$ a.e. in $B$, so $\nabla u_{n}(x) \rightarrow \nabla \bar{u}(x)$ a.e. in $B$. Thus

$$
\lim _{n \rightarrow \infty} \varrho\left(u_{n}-\bar{u}\right)=\int_{B} \lim _{n \rightarrow \infty}\left|\nabla u_{n}-\nabla \bar{u}\right|^{p(x)}+R(|x|) \lim _{n \rightarrow \infty}\left|u_{n}-\bar{u}\right|^{p(x)} d x=0 .
$$

But according to Lemma 1.6, we have $\left\|u_{n}-\bar{u}\right\|_{*} \check{p} \leq \varrho\left(u_{n}-\bar{u}\right)$, and thus $\left\|u_{n}-\bar{u}\right\|_{*} \rightarrow 0$. As a result,

$$
\left\|u_{n}-\bar{u}\right\|_{V}=\left\|u_{n}-\bar{u}\right\|_{*}+\left|u_{n}-\bar{u}\right|_{a, q(\cdot)}+\left|u_{n}-\bar{u}\right|_{b, r(\cdot)} \rightarrow 0,
$$

and thus $u_{n} \rightarrow \bar{u}$ strongly in $V$, as desired.

Lemma 2.6 Let $V=W_{0, \text { rad }}^{1, p(\cdot)}(B) \cap L_{a}^{q(\cdot)}(B) \cap L_{b}^{r(\cdot)}(B)$ and consider the functional $I: V \rightarrow \mathbb{R}$ defined by

$$
I(u):=\psi(u)-\varphi(u)
$$

where $\psi$ and $\varphi$ are as in (17) and (18), respectively. Then I has a nontrivial critical point in $K$.

Proof We show that $I$ satisfies the conditions of the MPG theorem. It is clear that $I(0)=0$. Take $e \in K$. Then it follows that

$$
I(t e) \leq M \frac{t^{p^{+}}}{p(x)}\|e\|_{*}^{\hat{p}_{*}}+\frac{t^{r^{+}}}{r(x)} \int_{B} b(x)|e|^{r(x)} d x-\frac{t^{q^{-}}}{q(x)} \int_{B} a(x)|e|^{q(x)} d x .
$$

Now let $p^{+}, r^{+}<q^{-}$. Then for $t$ large enough, $I(t e)$ is negative.

Condition (iii) of the MPG theorem is satisfied. Let $u \in \operatorname{Dom}(\psi)$ with $\|u\|_{V}=\rho>0$. Notice that by Lemma 2.4, for $u \in K$, we have

$$
\|u\|_{*} \geq \frac{\|u\|_{V}}{1+C 1+C_{2}}
$$

Also,

$$
\varphi(u)=\frac{1}{q(x)} \int_{B} a(x)|u|^{q(x)} d x \leq C_{3}|u|_{q(\cdot)}^{\tilde{q}} \leq C^{\tilde{q}} C_{3}\|u\|_{*}^{\tilde{q}} \leq C_{4}\|u\|_{V}^{\tilde{q}}=C_{4} \rho^{\tilde{q}} .
$$

where $C$ is the same as in Proposition 1.4(ii), and

$$
\tilde{q}= \begin{cases}q^{-}, & |u|_{p(\cdot)}<1, \\ q^{+}, & |u|_{p(\cdot)} \geq 1 .\end{cases}
$$

Relations (26) and (27) imply

$$
I_{K}(u) \geq \frac{\rho^{\check{p}}}{p^{+}\left(1+C_{1}+C_{2}\right)^{p^{+}}}-C_{4} \rho^{\tilde{q}}>0,
$$


where

$$
\check{q}= \begin{cases}q^{+}, & \|u\|_{*}<1, \\ q^{-}, & \|u\|_{*} \geq 1,\end{cases}
$$

provided that $\rho>0$ is small enough as $\check{p}=p^{+}<q^{-}=\tilde{q}$ and $C_{1}, C_{2}, C_{3}, C_{4}$ are positive constants. If $u \notin \operatorname{Dom}(\psi)$, then clearly $I(u)>0$.

Step 2. We show that the triple $\left(\psi_{K}, \varphi, K\right)$ satisfies the pointwise invariance condition at $u$ when $G=0$. To show this statement, we need following lemma.

Lemma 2.7 Let $p \in C_{+}(\bar{B})$, and let $p^{\prime}(x)=\frac{p(x)}{p(x)-1}$ be the conjugate exponent of $p(x)$. Let radial function $R: \mathbb{R} \rightarrow[0,+\infty)$ satisfy in one of the following conditions:

(i) $R(x)=\alpha$, where $\alpha>0$ is a real constant.

(ii) $R \in L^{\infty}(B)$ and $\mu>0$, where $\mu=\inf _{u \in W_{0}^{1, p(\cdot)}(B) \backslash\{0\}} \frac{\int_{B}|\nabla u|^{p(x)} d \xi}{\int_{B}|u|^{p(x)} d x}$.

Let $f: B \rightarrow \mathbb{R}$ be a continuous function. Suppose there exist $\alpha, \beta>0$ such that for any $u \in$ $L^{p(x)}(B)$,

$$
|f(u(x))| \leq \alpha+\beta|u(x)|^{p^{*}(x)-1}
$$

and

$$
f(u(x)) u(x) \leq 0
$$

Then for every $h \in L^{p^{\prime}(\cdot)}(B)$, the problem

$$
\begin{cases}-\Delta_{p(x)} u+R(x) u^{p(x)-2} u=f(u)+h(x), & x \in B, \\ u=0, & x \in \partial B\end{cases}
$$

admits at least one weak solution.

Proof First, notice that by integration respect to $u$ we can see that there exist $\alpha_{1}, \beta_{1}>0$ such that

$$
|F(u(x))| \leq \alpha_{1}+\beta_{1}|u(x)|^{p^{*}(x)}
$$

for all $u: B \rightarrow \mathbb{R}$ and $F(u(x)) \leq 0$ for all $u \in L^{p(x)}(B)$, where $F(t)=\int_{0}^{t} f(s) d s$. Now consider the following energy functional corresponding to problem $(28)$ on $W_{0}^{1, p(\cdot)}(B)$ :

$$
J(u)=\frac{1}{p(x)} \int_{B}|\nabla u|^{p(x)}+R(x)|u|^{p(x)} d x-\int_{B} F(u) d x-\int_{B} h u d x .
$$

By the Hölder inequality, Remark 1.5, and Lemma 1.6 we have

$$
J(u) \geq \frac{1}{p^{+}}\|u\|_{*}^{\check{p}}-C\|u\|_{*} .
$$


Since $p \in C(\bar{B})$, $J$ is coercive and clearly weakly lower semicontinuous on $W_{0}^{1, p(\cdot)}(B)$. So by the Weierstrass theorem (Theorem 1.9) $J$ has a global minimum point, which means that problem (28) admits at least one solution.

Lemma 2.8 Let $u \in \operatorname{Dom}(\psi)$. Then there exists $v \in \operatorname{Dom}(\psi)$ such that

$$
-\Delta_{p(x)} v+R(x) v^{p(x)-1}=a(x) u(x)^{q(x)-1}-b(x) v^{r(x)-1} .
$$

Proof Let $u \in \operatorname{Dom}(\psi)$, so that $0 \leq u \in K$. For $f(v(x))=-b(x)(v(x))^{r(x)-1}$, we can see that

$$
f(v(x)) v(x) \leq 0 \quad \text { and } \quad|f(v(x))| \leq C|v(x)|^{p^{*}(x)-1}
$$

so by Lemma 2.7 this problem admits at least one solution if $h(x)=a(x) u(x)^{q(x)-1}$ belongs to $L^{p^{\prime}(x)}(B)$. However, we have

$$
\int_{B}|h(x)|^{p^{\prime}(x)} d x \leq C \int_{B}|u(x)|^{p^{\prime}(x)(q(x)-1)} d x<+\infty .
$$

Since $p \in C_{+}(B)$ and $p(x)<q(x)$, we have $p^{\prime}(x)(q(x)-1)=\frac{p(x)}{p(x)-1}(q(x)-1)>1$ and also $a \in$ $L^{\infty}(B)$. The result is achieved.

\section{Acknowledgements}

The authors thank the anonymous reviewers for their careful reading of the manuscript and their insightful comments and suggestions.

\section{Funding}

This paper has been supported by the RUDN University Strategic Academic Leadership Program and Dipartimento di Matematica e Informatica - Universitá degli Studi di Catania. The first author is partially supported by I.N.D.A.M - P.R.I.N 2019.

\section{Availability of data and materials}

Data sharing not is applicable to this paper as no datasets were generated or analyzed during the current study.

\section{Competing interests}

The authors declare that they have no competing interests.

\section{Authors' contributions}

All authors discussed the results and implications and commented on the manuscript at all stages. All authors read and approved the final manuscript.

\section{Author details}

1 Dipartimento di Matematica e Informatica, Università di Catania, Catania, Italy. ${ }^{2}$ RUDN University, 6 Miklukho-Maklay St, 117198 Moscow, Russia. ${ }^{3}$ Department of Pure Mathematics, Imam Khomeini International University, 34149-16818 Qazvin, Iran.

\section{Publisher's Note}

Springer Nature remains neutral with regard to jurisdictional claims in published maps and institutional affiliations.

Received: 17 November 2020 Accepted: 6 April 2021 Published online: 21 April 2021

\section{References}

1. Rǎdulescu, V.D., Repoveš, D.D.: Partial Differential Equations with Variable Exponents, Variational Methods and Qualitative Analysis. Monographs and Research Notes in Mathematics. CRC Press, Boca Raton (2015)

2. Arora, R., Giacomoni, J., Warnault, G.: A Picone identity for variable exponent operators and applications. Adv. Nonlinear Anal. 9(1), 327-360 (2020)

3. Cowan, C., Razani, A.: Singular solutions of a p-Laplace equation involving the gradient. J. Differ. Equ. 269, 3914-3942 (2020). https://doi.org/10.1016/j.jde.2020.03.017

4. Cowan, C., Razani, A.: Singular solutions of a Lane-Emden system. Discrete Contin. Dyn. Syst. 41(2), 621-656 (2021). https://doi.org/10.3934/dcds.2020291 
5. Makvand Chaharlang, M., Ragusa, M.A., Razani, A.: A sequence of radially symmetric weak solutions for some nonlocal elliptic problem in $R^{n}$. Mediterr. J. Math. 17(2), 1-12 (2020)

6. Ragusa, M.A., Tachikawa, A.: Regularity for minimizers for functionals of double phase with variable exponents. Adv. Nonlinear Anal. 9, 710-728 (2020). https://doi.org/10.1515/anona-2020-0022

7. Ragusa, M.A., Razani, A.: Weak solutions for a system of quasilinear elliptic equations. Contrib. Math. 1, 11-16 (2020)

8. Razani, A.: Subsonic detonation waves in porous media. Phys. Scr. 94, 085209 (2019). https://doi.org/10.1088/1402-4896/ab029b

9. Safari, F., Razani, A.: Existence of positive radial solutions for Neumann problem on the Heisenberg group. Bound. Value Probl. 2020, 88 (2020). https://doi.org/10.1186/s13661-020-01386-5

10. Sarafi, F., Razani, A.: Nonlinear nonhomogeneous Neumann problem on the Heisenberg group. Appl. Anal. (2020). https://doi.org/10.1080/00036811.2020.1807013

11. Safari, F., Razani, A.: Existence of radial solutions of the Kohn-Laplacian problem. Complex Var. Elliptic Equ. (2020). https://doi.org/10.1080/17476933.2020.1818733

12. Safari, F., Razani, A.: Positive weak solutions of a generalized supercritical Neumann problem. Iran. J. Sci. Technol. Trans. A, Sci. 44(6), 1891-1898 (2020). https://doi.org/10.1007/s40995-020-00996-z

13. Shi, X., Rădulescu, V.D., Repoveš, D.D., Zhang, Q.: Multiple solutions of double phase variational problems with variable exponent. Adv. Calc. Var. 13(4), 384-401 (2020)

14. Zhang, Q., Rădulescu, V.D.: Double phase anisotropic variational problems and combined effects of reaction and absorption terms. J. Math. Pures Appl. 118(9), 159-203 (2018)

15. Karagiorgos, Y., Yannakaris, N.: A Neumann problem involving the $p(x)$-Laplacian with $p=\infty$ in a subdomain. Adv Calc. Var. 9(1), 65-76 (2016)

16. Kováčik, O., Rákosnik, J.: On spaces $\left.\right|^{p(x)}$ and $w^{k, p(x)}$. Czechoslov. Math. J. 41(4), 592-618 (1991)

17. Fan, X.L., Zhao, D.: On the generalized Orlicz-Sobolev space $w^{k, p(x)}(\omega)$. Gansu Jiaoyu Xueyuan Xuebao. Shehui Kexue Ban 12(1), 1-6 (1998)

18. Diening, L., Harjulehto, P., Hästö, P., Råužička, M.: Lebesgue and Sobolev Spaces with Variable Exponents. Lecture Notes in Mathematics. Springer, Heidelberg (2011)

19. Badiale, M., Serra, E.: Semilinear Elliptic Equations for Beginners, Existence Results via the Variational Approach. Springer, Cham (2010)

20. Ambrosetti, A., Rabinowitz, P.H.: Dual variational methods in critical point theory and applications. J. Funct. Anal. 14, 349-381 (1973). https://doi.org/10.1016/0022-1236(73)90051-7

21. Pólya, G., Szegö, G.: Problems and Theorems in Analysis I: Series. Integral Calculus. Theory of Functions. Springer, Berlin (1978)

22. Moameni, A., Salimi, L.: Existence results for supercritical Neumann problem with a convex-concave non-linearity. Ann. Mat. Pura Appl. 198, 1165-1184 (2019). https://doi.org/10.1007/s10231-018-0813-1

\section{Submit your manuscript to a SpringerOpen ${ }^{\circ}$ journal and benefit from:}

- Convenient online submission

- Rigorous peer review

- Open access: articles freely available online

- High visibility within the field

\section{- Retaining the copyright to your article}

